\title{
Preventing and managing hypertension: do not forget the night
}

\author{
Luis M. Ruilope ${ }^{1,2,3} \cdot$ Gema Ruiz-Hurtado ${ }^{1,2} \cdot$ Alejandro Lucia ${ }^{3,4}$
}

Received: 11 August 2021 / Revised: 13 August 2021 / Accepted: 19 August 2021 / Published online: 15 September 2021

(c) The Japanese Society of Hypertension 2021

Most functions and responses of the body vary over the course of a day-known as "circadian rhythm", a crucial factor for survival. The circadian rhythm is controlled by the central biological clock in the suprachiasmatic nucleus, which has evolved to synchronize our daily activities based on signals from the external environment, such as light (or darkness) exposure. Notably, blood pressure (BP) has a marked circadian pattern, with a normal drop of $>10 \%$ observed from daytime to nighttime (i.e., "dipping pattern"), and dysregulation of this usual pattern is associated with a higher risk for cardiovascular disease (CVD) and hypertension [1]. The influence of circadian rhythm on CVD risk is indeed reflected not only by the fact that $24 \mathrm{~h}$ ambulatory BP monitoring is a stronger prognostic factor for CVD and mortality than clinic ("office") BP [2] but also by the clinical importance of nighttime ambulatory BP [3], even beyond that of daytime BP [4]. Thus, nondipper $(<10 \%)$ [5] or riser patterns (i.e., increase in BP from daytime to nighttime) [6] as well as morning BP surge [7] are strong predictors of adverse events.

Sleeping is the main nighttime activity. Although some of its biological roles remain to be elucidated, sleep is essential for many vital functions, including development, energy conservation, cognitive and physical performance, and immune function. The total daily sleep duration in the first humans was $>8 \mathrm{~h}$ during most of the year, with some seasonal variationsincreases and decreases during shorter (winter) and longer (summer) natural photoperiods, respectively [8]. However,

Luis M. Ruilope

ruilope@icloud.com

1 Cardiorenal Translational Laboratory, Institute of Research i+12, Hospital Universitario 12 de Octubre, Madrid, Spain

2 CIBER-CV, Hospital Universitario 12 de Octubre, Madrid, Spain

3 Faculty of Sport Sciences, Universidad Europea de Madrid, Madrid, Spain

4 Research Institute of the Hospital Universitario 12 de Octubre i+12, Madrid, Spain individuals in Western societies are currently exposed to numerous sleep disruptors, such as shift work, multiple jobs, the internet, television, and especially alterations of the natural light-dark cycle. The latter is due to the wide availability of artificial light (especially of the blue spectrum), attenuated exposure to daylight within buildings, and evening use of light_emitting devices, all of which decrease the strength of natural light_dark signals that entrain circadian systems $[8,9]$.

Not surprisingly, sleep disturbances are prevalent among westerners (e.g., affecting one-third of the U.S. adult population [10]). This is an important problem because sleep disturbances can have a negative impact on health status, including cardiovascular health. Recent meta-analytical evidence indicated that not only a short ( $<7 \mathrm{~h}$ ) but also (and especially) a long ( $>8 \mathrm{~h}$ ) sleep duration is associated with a higher risk of mortality and CVD events [11]. Likewise, sleep quality (i.e., the self-reported, retrospective assessment of the sleep experience) is important, as it is inversely associated with the incidence of coronary heart disease-albeit it does not seem to be linked with mortality or other CVD outcomes such as stroke or mortality [11].

The disruption of the natural light-dark cycle that characterizes modern societies can contribute to sleepmaintenance insomnia (or simply "insomnia")—a condition characterized by difficulty staying asleep, nocturnal awakenings and, in particular, waking too early and struggling to get back to sleep. Insomnia is the most common sleep disorder, affecting $\sim 30 \%$ of the general population, and is associated with a wide cluster of CVDs and metabolic disorders, including hypertension [12]. Of major importance is obstructive sleep apnea (OSA), a breathing disorder characterized by repeated episodes of complete or partial upper_airway occlusion (and subsequent arterial hypoxemia) leading to sustained activation of carotid body chemoreceptors that reflexively upregulate sympathetic nervous system activity [13]. OSA is the most common condition associated with resistant hypertension, and it is found in $30-40 \%$ of all patients with hypertension [14]. 
In a topical study recently published in this journal, Kario et al. reported the association between self-reported sleep duration and nighttime BP in 2236 patients who were followed for $\sim 7 \mathrm{y}$ on average [15]. Short sleep duration (<6 h/night, vs. normal ["optimal"] duration [6-9 h/night]) was significantly associated with a more than twofold higher risk of atherosclerotic CVDs, especially stroke. An important finding of their study was that even 'shorter sleepers' with well-controlled nighttime systolic BP (i.e., $<120 \mathrm{mmHg}$ ) had a more than threefold higher risk of atherosclerotic CVD and coronary artery disease. Conversely, "optimal sleepers" were also at significantly higher risk of stroke if nighttime systolic BP was uncontrolled. These findings therefore reflect the importance of ensuring not only adequate control of nocturnal hypertension but also healthy sleep to reduce the risk of CVD, especially stroke. In this context, sleeping pills are frequently prescribed to patients with arterial hypertension and can contribute to the improvement of both sleep and BP control [16]. For instance, a nonhypotensive sedative, zolpidem, could be useful in patients with poor-quality sleep and nighttime hypertension who exhibit a nondipper pattern due to the capacity of this drug to improve sleep quality and stress status, converting nondippers with poor sleep quality into dippers [17].

In summary, both nighttime BP and sleep are important variables to be considered and monitored by those involved in the prevention and management of hypertension. Interventional research is needed to determine how to improve both components.

\section{Compliance with ethical standards}

Conflict of interest The authors declare no competing interests.

Publisher's note Springer Nature remains neutral with regard to jurisdictional claims in published maps and institutional affiliations.

\section{References}

1. Douma LG, Gumz ML. Circadian clock-mediated regulation of blood pressure. Free Radic Biol Med. 2018;119:108-14.

2. Dolan E, Stanton A, Thijs L, Hinedi K, Atkins N, McClory S, et al. Superiority of ambulatory over clinic blood pressure measurement in predicting mortality: the Dublin outcome study. Hypertension. 2005;46:156-61.
3. Yang WY, Melgarejo JD, Thijs L, Zhang ZY, Boggia J, Wei FF, et al. Association of office and ambulatory blood pressure with mortality and cardiovascular outcomes. JAMA. 2019;322: 409-20.

4. Fagard RH, Celis H, Thijs L, Staessen JA, Clement DL, De Buyzere ML, et al. Daytime and nighttime blood pressure as predictors of death and cause-specific cardiovascular events in hypertension. Hypertension. 2008;51:55-61.

5. Salles GF, Reboldi G, Fagard RH, Cardoso CR, Pierdomenico SD, Verdecchia P, et al. Prognostic effect of the nocturnal blood pressure fall in hypertensive patients: the ambulatory blood pressure collaboration in patients with hypertension $(\mathrm{ABC}-\mathrm{H})$ meta-analysis. Hypertension. 2016;67:693-700.

6. Kario K, Hoshide S, Mizuno H, Kabutoya T, Nishizawa M, Yoshida T, et al. Nighttime blood pressure phenotype and cardiovascular prognosis: practitioner-based nationwide JAMP study. Circulation. 2020;142:1810-20.

7. Hoshide S, Kario K, de la Sierra A, Bilo G, Schillaci G, Banegas $\mathrm{JR}$, et al. Ethnic differences in the degree of morning blood pressure surge and in its determinants between Japanese and European hypertensive subjects: data from the ARTEMIS study. Hypertension. 2015;66:750-6.

8. de la Iglesia HO, Moreno C, Lowden A, Louzada F, Marqueze E, Levandovski R, et al. Ancestral sleep. Curr Biol. 2016;26: R271-R272.

9. Valenzuela PL, Carrera-Bastos P, Gálvez BG, Ruiz-Hurtado G, Ordovas JM, Ruilope LM, et al. Lifestyle interventions for the prevention and treatment of hypertension. Nat Rev Cardiol. 2021; $18: 251-75$.

10. Liu Y, Wheaton A, Chapman D, Cunningham T, Lu H, Croft J. Prevalence of healthy sleep duration among adults-United States, 2014. MMWR Morb Mortal Wkly Rep. 2016;65: $137-41$.

11. Kwok CS, Kontopantelis E, Kuligowski G, Gray M, Muhyaldeen A, Gale CP, et al. Self-reported sleep duration and quality and cardiovascular disease and mortality: a dose-response meta-analysis. J Am Heart Assoc. 2018;7:e008552.

12. Stavropoulos K, Imprialos KP, Doumas M, Karagiannis A, Papademetriou V. Insomnia and hypertension: a misty landscape. J Clin Hypertens. 2019;21:835-7.

13. Dewan NA, Nieto FJ, Somers VK. Intermittent hypoxemia and OSA: implications for comorbidities. Chest. 2015;147:266-74.

14. Pedrosa RP, Drager LF, Gonzaga CC, Sousa MG, de Paula LK, Amaro AC, et al. Obstructive sleep apnea: the most common secondary cause of hypertension associated with resistant hypertension. Hypertension. 2011;58:811-7.

15. Kario K, Hoshide S, Nagai M, Okawara Y, Kanegae H Sleep and cardiovascular outcome in relation to nocturnal hypertension: the J-HOP nocturnal blood pressure study. Hypertens Res. 2021. https://doi.org/10.1038/s41440-021-00709-y.

16. Tan X, van Egmond L, Chapman CD, Cedernaes J, Benedict C. Aiding sleep in type 2 diabetes: therapeutic considerations. Lancet Diabetes Endocrinol. 2018;6:60-8.

17. Huang Y, Mai W, Cai X, Hu Y, Song Y, Qiu R, et al. The effect of zolpidem on sleep quality, stress status, and nondipping hypertension. Sleep Med. 2012;13:263-8. 